\title{
Informe de caso: tumor de estroma gastrointestinal mesentérico (EGIST)
}

\author{
Case report: mesenteric gastrointestinal stromal tumor \\ (EGIST) \\ Pesantez Brito Ismael Francisco', Ordoñez Velecela María Soledad², \\ Oyervide Soto Juan Manuel', García Cayamcela Ana Belén', Men- \\ dieta Bermeo Edgar Gustavo³.
}

VOLUMEN 37 | N 2 | SEPTIEMBRE 2019

FECHA DE RECEPCIÓN: 14/05/2019 FECHA DE APROBACIÓN: 08/08/2019 FECHA PUBLICACIÓN: $\quad$ 10/09/2019

1. Libre ejercicio

2. Universidad de Cuenca

3. Hospital José Carrasco Arteaga

\begin{tabular}{r|l} 
Caso & Clinical \\
Clínico & Case
\end{tabular}

DOI: https://doi.org/10.18537/RFCM.37.02.08

Correspondencia:

ismael.fpb囚gmail.com

Dirección:

Eugenio Espejo 507 y Miguel Cordero

Código Postal:

010101

Teléfono:

07-2823343 / 0984549206

Cuenca - Ecuador

\section{RESUMEN}

Los tumores del estroma extragastrointestinal son neoplasias mesenquimatosas raras que pueden presentarse en epiplón y mesenterio. Se presenta el caso clínico de un paciente cuencano de la tercera edad con cuadro clínico caracterizado por dolor, distensión abdominal, falta de eliminación de gases y heces de $24 \mathrm{~h}$ de evolución, al examen físico se evidenció abdomen distendido, dolor a la palpación en forma difusa, ruidos hidroaéreos aumentados. Fue intervenido quirúrgicamente, encontrándose un tumor a $80 \mathrm{~cm}$ de la válvula ileocecal dependiente de mesenterio, de aproximadamente $7 \times 6 \mathrm{~cm}$ que comprometía asas intestinales; histopatología reportó un tumor del estroma extra gastrointestinal CD 117 positivo; paciente evoluciono favorablemente, se inició deambulación a las 24 horas y dieta líquida a las 72 horas con buena tolerancia, alta médica al quinto día postquirúrgico. Inicio tratamiento oncológico a los 4 meses posteriores a la cirugía con Imatinib $400 \mathrm{mg}$, actualmente paciente con evolución favorable sin recurrencia.

Palabras Clave: informes de casos, tumores del estroma gastrointestinal, neoplasias abdominales, procedimientos quirúrgicos operativos.

\section{ABSTRACT}

Extragastrointestinal stromal tumors are rare mesenchymal neoplasms that can occur in the omentum and mesentery. A clinical case of an elderly person from Cuenca is presented, the patient has symptoms like: pain, abdominal distension, lack of elimination of gases and feces of 24 hours of evolution, the physical examination showed a distended abdomen, diffuse palpation pain, increased hydro noise.

The patients was surgically intervened, a tumor was found $80 \mathrm{~cm}$ from the mesoenterium-dependent ileocecal valve, approximately $7 \times 6 \mathrm{~cm}$ that involved intestinal loops; histopathology reported a CD 117 positive extra gastrointestinal stromal tumor; patient evolved favorably, ambulation began at 24 hours and liquid diet at 72 hours with good tolerance, medical discharge was decided on the fifth day after surgery. Start oncological treatment at 4 months after surgery with imatinib $400 \mathrm{mg}$, currently a patient with favorable evolution without recurrence. 
Keywords: case reports, gastrointestinal stromal tumors, abdominal neoplasms, surgical procedures operative.

\section{INTRODUCCION}

Las neoplasias de estroma gastrointestinal (GIST),tienen una incidencia a nivel mundial $14-20$ por millón de habitantes al año [1]. La edad media de presentación es alrededor de los 60 años, sin diferencia de sexo, aunque algunos estudios han concluido un predominio mayor en hombres. Afecta a todo el tracto gastrointestinal, pudiendo presentarse extraintestinalmente en menos del $1 \%$ de los casos $[2,3]$.

Las neoplasias de estroma extra gastrointestinal (EGIST), son neoplasias raras, que representan menos del $5 \%$ de todos los GISTs, de los cuales aproximadamente el $80 \%$ se encuentran en el epiplón o mesenterio [4]. Se han descrito tres hipótesis para su desarrollo: la primera afirma que tienen un origen en el tracto digestivo con un desarrollo exofítico y posterior adquisición de autonomía; la segunda apoya que los EGIST son metástasis peritoneales de un GIST no diagnosticado; y la tercera propone un origen mesotelial con características similares a las células de Cajal [5].

El $90 \%$ de los GITS primarios pueden tener mutaciones en los genes KIT (80\%) lo cual da positividad para el anticuerpo monoclonal CD-11794-95\% de los casos o PDGFRA (10\%), mientras que el restante $10 \%$ no tiene mutación en ninguno de los genes por lo que se denomina GIST salvaje [6].

\section{CASO CLINICO}

Paciente de 72 años de edad, ocupación agricultor, residencia y procedencia Cuenca, con antecedentes de hipertensión arterial, colecistectomía convencional, prostatectomía y hernioplastia inguinal bilateral. Acude a emergencia tras presentar distensión y dolor abdominal de 12 horas de evolución sin causa aparente, falta de eliminación de gases y heces, acompañado de náuseas que llevan al vómito por dos ocasiones de tipo alimenticio.

Al examen físico signos vitales estables, en abdomen se evidencia gran distensión, dolor a la palpación superficial y profunda dolor difuso, hipertimpanismo, ruidos hidroaéreos aumentados en hemiabdomen superior y disminuidos en el inferior, al tacto rectal no se evidencia contenido.
En los exámenes complementarios se encontró, biometría hemática leucocitos de 17,62 103 $\mu \mathrm{l}$ con predominio de los neutrófilos $93 \%$ hemoglobina $15,5 \mathrm{~g} / \mathrm{dl}$ plaquetas $238 \times 10^{3}$, tiempo de protrombina $12,2 \mathrm{seg}$ tromboplastina $26,7 \mathrm{seg}$. Se solicitó una radiografía de abdomen de pie en la que se observó niveles hidroaéreos y ausencia de gas en la ampolla rectal. En la tomografía simple y contrastada de abdomen se detectó a nivel de la bifurcación de las ilíacas, un proceso ocupativo heterogéneo con áreas de necrosis central que medía $79 \times 63 \mathrm{~mm}$, localizada en el mesenterio a nivel de fosa y flanco derecho, que desplazaba las asas intestinales ileales y el colon hacia la porción distal. Con estos resultados se decide complementar el diagnóstico con marcadores tumorales (Antígeno carcinoembrionario, CA 19-9 y alfa fetoproteína) con parámetros dentro de lo normal.

\section{EVOLUCION}

Paciente es hospitalizado con cuadro clínico de abdomen agudo oclusivo por masa abdominal en estudio, se mantuvo hemodinámicamente estable, afebril, se colocó sonda nasogástrica la cual da un débito de 1500 cc aproximadamente de contenido intestinal mejorando parcialmente la distensión abdominal y el dolor. Se solicitó un ultrasonido abdominal, en el cual se evidencia una masa hipoecoica sin flujo al doppler (Imagen № 1). Veinte y cuatro horas después del manejo con sonda nasogástrica y reposición hídrica presenta mejoría y se decide realizar tránsito esófago-gastro-intestinal donde se encuentra una oclusión intestinal parcial asociada al proceso ocupativo (Imagen $\mathrm{N}^{\circ} 2$ ). 


$$
\text { Imagen } N^{\circ} 1
$$

Ultrasonido abdominal

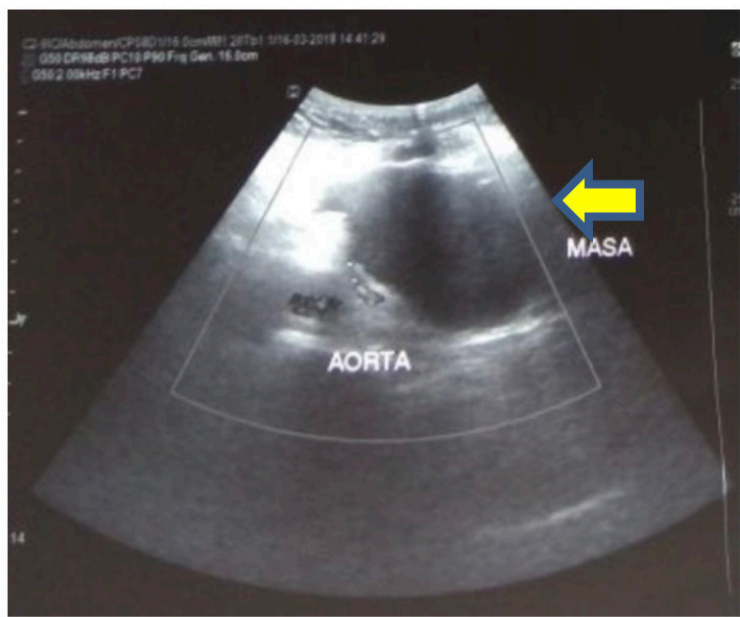

\section{Imagen $\mathrm{N}^{\circ} 2$}

Tránsito intestinal

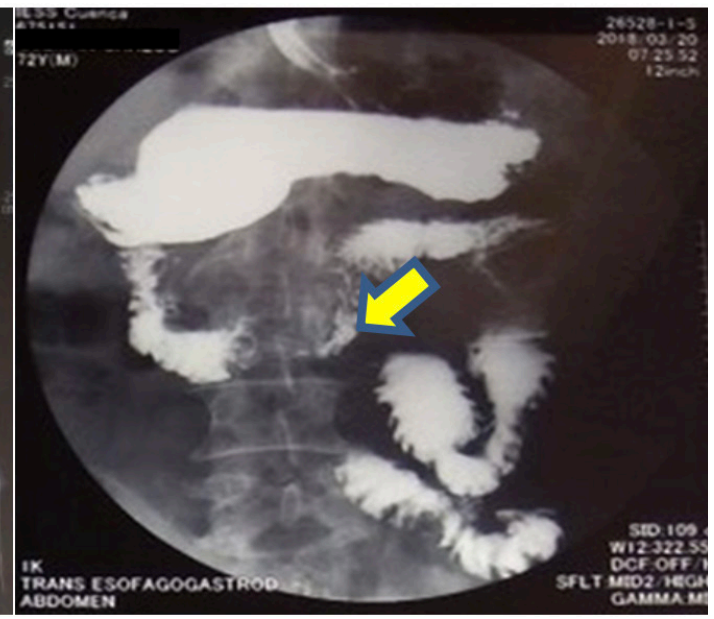

Fuente: Departamento de Imagenología Hospital José Carrasco Arteaga Elaborado por: Autores

Con estos resultados se complementa con una resonancia magnética abdominal, la misma que se observa masa de $7 \times 6 \mathrm{~cm}$ a nivel de flanco y fosa iliaca derecha sin invasión a estructuras vasculares importantes (Imagen $\mathrm{N}^{\circ} 3$ ).

\section{Imagen $N^{\circ} 3$}

Resonancia Magnética Abdominal: se evidencia masa, sin invasión a estructuras vasculares.

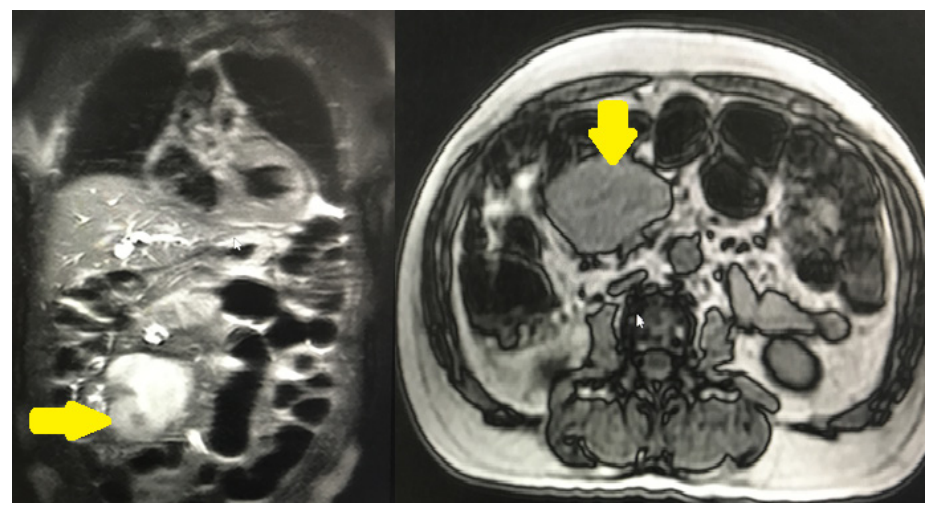

Fuente: Departamento de Imagenología Hospital José Carrasco Arteag Elaborado por: autores

Se decide realizar una laparoscopía exploratoria, observándose un tumor dependiente del mesenterio a $80 \mathrm{~cm}$ de la válvula ileocecal (Imagen $N^{\circ} 4$ ), debido al tamaño del mismo se decidió convertir a cirugía abierta. 


\section{Imagen $\mathrm{N}^{\circ} 4$}

Tumor dependiente del mesenterio observado durante la laparoscopia exploratoria.

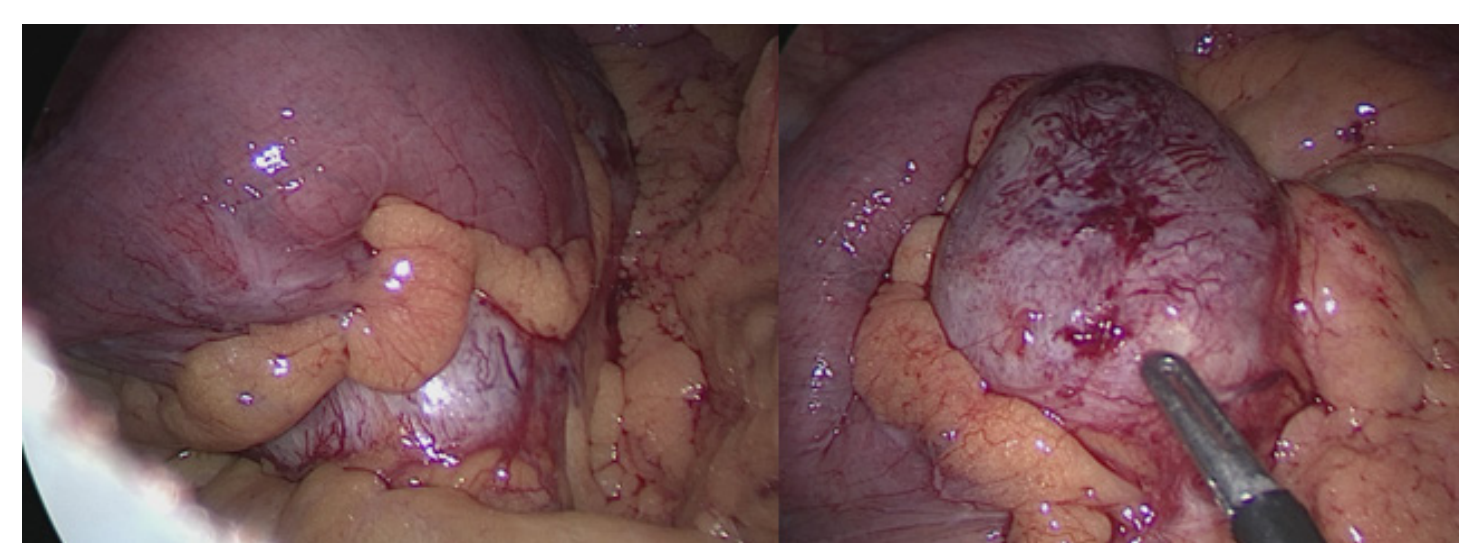

Fuente: Centro Quirúrgico Hospital José Carrasco Arteaga

Elaborado por: autores

La masa tumoral de $7 \times 6 \mathrm{~cm}$ fue resecada en bloque junto con una porción de intestino delgado la cual comprometía su vascularidad y se hallaba adherido. Se restableció la continuidad intestinal mediante una anastomosis íleo-ileallátero-lateral, sin complicaciones. El paciente permaneció hospitalizado durante 5 días, a las 24 horas inicio deambulación sin dificultad, la dieta inicio a las 72 horas con buena tolerancia posterior a la cirugía siendo dado de alta al quinto día de hospitalización.

\section{Imagen $\mathrm{N}^{0} 5$}

Con tinción de HE se observa neoplasia moderadamente diferenciada, constituida por elementos fusiformes con escasa atipia.

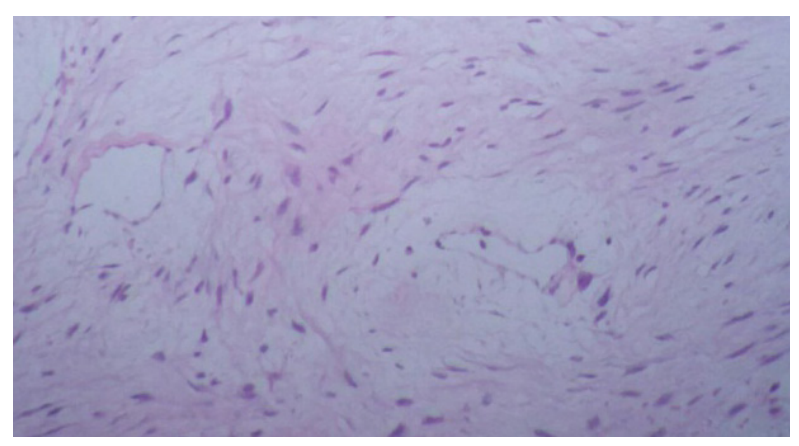

Fuente: Departamento de Anatomía Patológica Hospital José Carrasco Arteaga

Elaborado por: autores
El estudio histopatológico reportó (Imagen 5-6-7-8): tumor del estroma gastrointestinal (GIST) fusiforme primario demesenterio con compromiso de intestino, de bajo grado con conteo mitótico: $<5 / 5 \mathrm{~mm}$, de bordes libres y ganglios peritumorales libres de neoplasia. Con este resultado se refirió a paciente a oncología para inicio de tratamiento adyuvante con imatibid $400 \mathrm{mg}$, el cual se inició a los 4 meses posteriores al alta. Presento toxicidad caracterizada por nausea, diarrea y astenia, las cuales fueron disminuyendo en el transcurso del tratamiento. Actualmente paciente se encuentra estable sin recurrencia.

\section{Imagen $N^{\circ} 6$}

A mayor aumento, se observan los núcleos ahusados dispuestos en fascículos laxos. No se identifican figuras mitósicas.

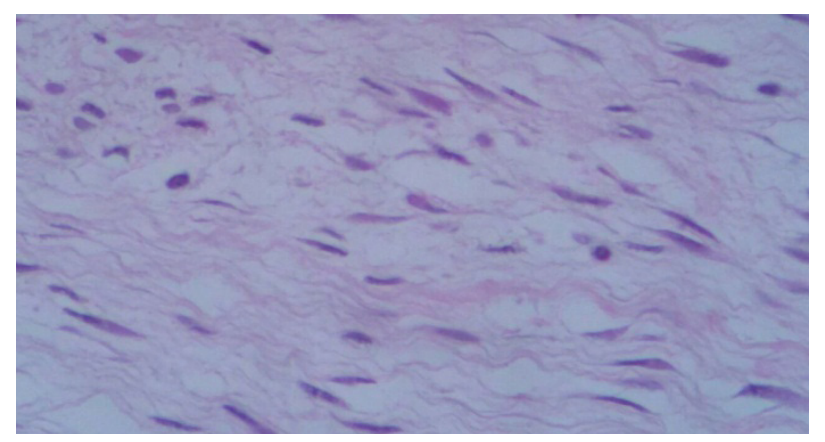

Fuente: Departamento de Anatomía Patológica Hospital José Carrasco Arteaga

Elaborado por: autores 


\section{Imagen $N^{0} 7$}

Todas las células expresan intensamente Vimentina lo que confirma la naturaleza mesenquimal de la lesión.

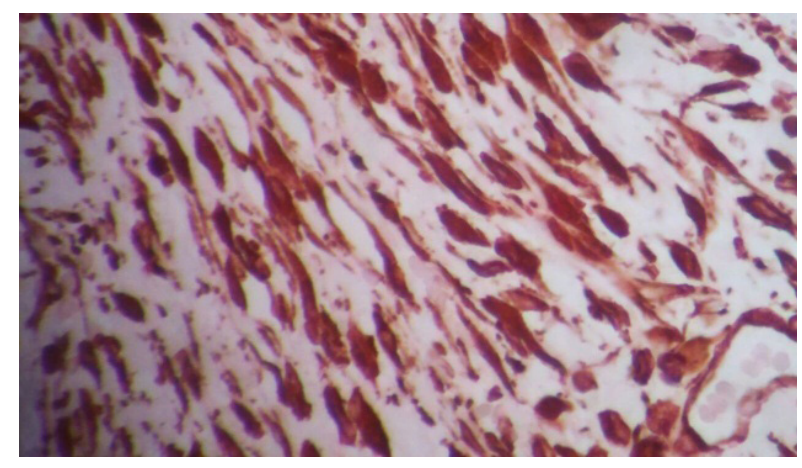

Fuente: Departamento de Anatomía Patológica Hospital José Carrasco Arteaga

Elaborado por: autores

\section{DISCUSIÓN}

Los EGISTS son neoplasias raras de origen mesenquimático derivadas de las células intersticiales de Cajal, suelen presentarse entre los 55 a 65 años, sin evidenciarse predominio por algún sexo [7].

Su presentación clínica puede debutar con síntomas que incluyen hemorragia gastrointestinal con su subsecuente anemia, saciedad temprana, distensión abdominal, dolor e incomodidad debido a la compresión del tumor. La presentación de síntomas va a depender del tamaño del tumor, tumores $<3 \mathrm{~cm}$ no producen sintomatología y muchas veces son hallazgos durante autopsias. Se ha visto que la diseminación linfática de los GIST no es común, y por lo general las metástasis suelen ser a hígado u otros órganos del abdomen [8].

El diagnóstico se logra mediante intervenciones de imagen que pueden incluir ultrasonido abdominal en el cual se puede visualizar masas hipoecogénicas, que dependiendo del tamaño de las masas pueden desplazar estructuras vecinas y mostrar áreas quísticas, necróticas o hemorrágicas; la tomografía computarizada trifásica resonancia magnética, son la primera opción para estudiar ubicación y extensión tumoral. Hay pruebas que pueden ser útiles en la estatificación como la tomografía por emisión de positrones; la ecografía endoscópica con biopsia por aspiración con aguja fina es útil en la detección de GIST en el tracto gastrointestinal superior

\section{Imagen $N^{0} 8$.}

El marcador CD117 es claramente positivo en algunas células tumorales, sustentando el diagnóstico de tumor del estroma gastrointestinal (GIST)

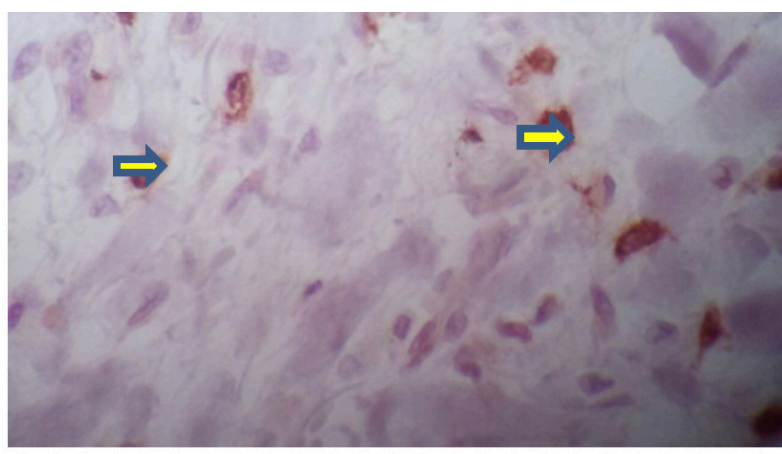

Fuente: Departamento de Anatomía Patológica Hospital José Carrasco Arteaga

Elaborado por: autores

porque la mayoría de tumores surgen debajo de la capa mucosa y crecen de forma endofítica [9].

El pilar fundamental del tratamiento es la cirugía, para los GIST localizados. La resección quirúrgica completa se la realiza cuando el tumor está localizado y es resecable, la disección de los ganglios regionales no es necesaria debido a que los GITS no provocan diseminación linfática [10].

Las indicaciones para la cirugía son tumores de 2 $\mathrm{cm}$ o más, crecimiento rápido con signos de malignidad, margen irregular, ulceración con hemorragia, cambios quísticos, necrosis y ecogenicidad heterogénea. En los GIST no gástricos se recomienda realizar resección independientemente del tamaño del tumor [11].

Los criterios radiológicos para irresecabilidad incluyen infiltración del tronco celiaco, la arteria mesentérica superior, vena mesentérica o porta. EI objetivo es lograr una cirugía de tipo R0 (cirugía optima) eliminar completamente el tumor dejando la capsula intacta, la resección segmentaria de intestino o estomago es aceptado sin ser necesario una resección agresiva ni extensa para extirpar el tejido no afectado, se deben examinar las superficie peritoneal y hepática. La resección R1 (resección marginal que contiene células tumorales) se la debe realizar siempre y cuando no implique secuelas funcionales, de preferencia se debería hacer un seguimiento del paciente antes de realizar 
una cirugía agresiva con daño permanente. Las indicaciones para la cirugía son tumores de $2 \mathrm{~cm}$ o más, crecimiento rápido con signos de malignidad, margen irregular, ulceración con hemorragia, cambios quísticos, necrosis y ecogenicidad heterogénea. En los GIST no gástricos se recomienda realizar resección independientemente del tamaño del tumor. Los objetivos de la cirugía deben incluir resección completa con márgenes negativos macroscópica y microscópicamente [12].

El tratamiento quirúrgico debe ser complementado con terapia adyuvante la cual tiene como objetivo reducir o retrasar el crecimiento de tumores microscópicos después de la resección completa de un GIST, el fármaco de elección es el Imatinib durante 3 años, el cual en varios estudios aleatorizados ha demostrado prolongar significativamente la supervivencia sin recidiva en los pacientes con GIST primario después de una resección curativa. El tratamiento neoadyuvante con imatinib debe considerarse para GIST localizados no metastásicos, cuando la resección R0 no es factible o para preservar la función de los órganos, se deberá realizar una evaluación temprana mediante tomografía computarizada o PET scan luego del primer mes de tratamiento con imatinib. El tratamiento neoadyuvante preoperatorio es de 4 a 6 meses [13]. El imatinib coadyuvante debe iniciarse tan pronto se inicie la vía oral. The National Comprehensive Cancer Network recomienda el tratamiento con imatinib antes y después de la cirugía de al menos 3 años para GIST de alto riesgo. La dosis recomendada es de 400mg vía oral al día [14].

El pronóstico luego de la cirugía depende de la tasa mitótica, tamaño del tumor, sitio del tumor, márgenes quirúrgicos y estado de ruptura del tumor de esto dependerá el riesgo que presente el paciente para la recidiva. Danielle A. Bischof y colaboradores determinaron que el tiempo que los pacientes permanecieron libres de enfermedad luego de resección quirúrgica y tratamiento adyuvante fue de $82 \%$ al 1 año, $89 \%$ a los 3 años y $92 \%$ a los 5 años [15].

\section{CONCLUSION}

Los EGIST son una patología poco frecuente y de difícil diagnóstico por lo que se necesita un manejo integral por un equipo multidisciplinario.

Los estudios de imagen permiten identificar esta patología de manera temprana y evaluar la resecabilidad del tumor.
La cirugía es un paso fundamental y que brinda al paciente un pronóstico favorable cuando se diagnostica de forma temprana el apoyo neoadyuvante o adyuvante obedecerá al reporte patológico.

\section{RECOMENDACIONES}

Aunque este tipo de neoplasias son raras deben ser consideradas entre las posibilidades diagnósticas, cuando el tumor presente las características orientativas que se han mencionado previamente; un oportuno diagnóstico e intervención mejora de forma notoria el pronóstico de los pacientes que padecen de esta neoplasia.

\section{ABREVIATURAS}

GIST: neoplasia del estroma gastro intestinal.

EGIST: neoplasia del estroma extra gastrointestinal.

HE: Hemotoxilina - Eosina.

\section{ASPECTOS BIOÉTICOS}

Los autores cuentan con el consentimiento informado por parte del paciente para la publicación del caso clínico y sus imágenes.

\section{INFORMACIÓN DE LOS AUTORES}

- Pesantez Brito Ismael Francisco. Especialista en Cirugía General. Libre Ejercicio. Cuenca Azuay - Ecuador.

e-mail: ismael.fpb@gmail.com

ORCID: https://orcid.org/0000-0001-7275-562X

- Ordoñez Velecela María Soledad. Médico General. Universidad de Cuenca. Posgrado de Cirugía General. Cuenca - Azuay - Ecuador. e-mail: msoledad_ordonez@hotmail.com ORCID: https://orcid.org/0000-0002-1254-0595

- Oyervide Soto Juan Manuel. Médico General. Libre ejercicio. Cuenca - Azuay - Ecuador. e-mail: juanoyervide@gmail.com.

ORCID: https://orcid.org/0000-0002-3037-0665

- García Cayamcela Ana Belén. Especialista en Cirugía General. Libre ejercicio. Cuenca - Azuay - Ecuador.

e-mail: a-belen-g@hotmail.com ORCID: https://orcid.org/0000-0001-7877-8021

- Mendieta Bermeo Edgar Gustavo. Especialista en Cirugía / Magister en Investigación. Hospital 
José Carrasco Arteaga. Cirugía. Emergencia. Cuenca - Azuay - Ecuador. e-mail: menedgar@gmail.com ORCID: https://orcid.org/0000-0003-0953-8862

\section{CONTRIBUCIÓN DE LOS AUTORES}

Los autores declaran haber contribuido de manera similar en la concepción, diseño, análisis e interpretación de los datos, redacción del manuscrito y aprobación de la versión final, así como estar en capacidad de responder de todos los aspectos del mismo.

\section{CONFLICTO DE INTERERES}

Los autores declaran no tener conflicto de intereses.

\section{FUENTES DE FINANCIAMIENTO}

Autofinanciad.

\section{REFERENCIAS BIBLIOGRÁFICAS}

1. Kays JK, Sohn JD, Kim BJ, Goze K, Koniaris LG. Approach to wild-type gastrointestinal stromal tumors. Transl Gastroenterol Hepatol. 2018;3:92-92.

2. Pandit N, Das GP, Dahal M, Awale L. An unexpected extra-gastrointestinal stromal tumor (E-GIST) on the jejunal mesentery. J Surg Case Rep. 2018;2018(12):1-3.

3. Zhao X, Yue C. Gastrointestinal stromal tumor. J Gastrointest Oncol. 2012;3(3):189.

4. Sousa D, Allen M, Mateus A, Cruz A, Marinho D, Ferreira A, et al. EGIST: a Rare Tumor. J Gastrointest Cancer. 2018;49(2):182-5.

5. Nishida T. Asian consensus guidelines for gastrointestinal stromal tumor: what is the same and what is different from global guidelines. Transl Gastroenterol Hepatol. 2018;3:11-11.

6. Board PATE. Gastrointestinal Stromal Tumors Treatment (PDQ $®)$ [Internet]. National Cancer Institute (US); 2011 [citado el 29 de abril de 2018]. Disponible en: https:// www.ncbi.nlm.nih.gov/books/NBK65712/

7. Gordillo R, Beltrán J, Díaz M. Tumor del estroma extra gastrointestinal (E-GIST) gigante de omento. Rev Chil Cir. 2013;65(2):166-171.

8. Dimofte M-G, Porumb V, Ferariu D, Bar N-C, Lunca S. EGIST of the greater omentumcase study and review of literature. Rom J Morphol Embryol. 2016;57(1):253-8.

9. Chen $\mathrm{T}, \mathrm{Xu} \mathrm{L}$, Dong $\mathrm{X}$, Li $\mathrm{Y}, \mathrm{Yu}$ J, Xiong $\mathrm{W}$, et al. The roles of CT and EUS in the preoperative evaluation of gastric gastrointestinal stromal tumors larger than 2 $\mathrm{cm}$. Eur Radiol [Internet]. 2019 [citado el 20 de enero de 2019]; Disponible en: http://link. springer.com/10.1007/s00330-018-5945-6

10. Koo D-H, Ryu M-H, Kim K-M, Yang $\mathrm{H}-\mathrm{K}$, Sawaki A, Hirota $\mathrm{S}$, et al. Asian Consensus Guidelines for the Diagnosis and Management of Gastrointestinal Stromal Tumor. Cancer Res Treat Cancer Res Treat. 2016;48(4):1155-66.

11. Dimofte M-G, Porumb V, Ferariu D, Bar N-C, Lunca S. EGIST of the greater omentumcase study and review of literature. Rom J Morphol Embryol. 2016;57(1):253-8.

12. Nishida T, Blay J-Y, Hirota S, Kitagawa $Y$, Kang Y-K. The standard diagnosis, treatment, and follow-up of gastrointestinal stromal tumors based on guidelines. Gastric Cancer. 2016;19(1):3-14.

13. Poveda A, García del Muro X, LópezGuerrero JA, Cubedo R, Martínez V, Romero I, et al. GEIS guidelines for gastrointestinal sarcomas (GIST). Cancer Treat Rev. 2017;55:107-19.

14. Bischof $D A$, Dodson $R$, Jimenez MC, Behman R, Cocieru A, Blazer DG, et al. Adherence to Guidelines for Adjuvant Imatinib Therapy for GIST: A Multi-institutional Analysis. J Gastrointest Surg. 2015;19(6):1022-8.

15. Bischof DA, $\operatorname{Kim} Y$, Dodson $R$, Jimenez $M C$, Behman R, Cocieru A, et al. Conditional Disease-Free Survival After Surgical Resection of Gastrointestinal Stromal Tumors: A Multi-institutional Analysis of 502 Patients. JAMA Surg. 2015;150(4):299. 